\title{
MODELING OF FRICTIONS IN THE TRANSMISSION ELEMENTS OF A ROBOT AXIS FOR ITS IDENTIFICATION
}

\author{
Gabriel Abba ${ }^{*, 1}$ Philippe Sardain ${ }^{* *, 1}$ \\ ${ }^{*}$ LGIPM, École Nationale d'ingénieurs de Metz, Ile du \\ Saulcy, 57045 METZ Cedex, France, abba@enim.fr \\ ** LMS, Université de Poitiers, SP2MI, 86962 \\ Futuroscope Cedex, sardain@lms.univ-poitiers.fr
}

\begin{abstract}
Control in robotics needs more and more precise models of the mechanical parts of the structure and specially for a complex system such as a biped robot. An important but difficult aspect of this work is the modeling of the mechanical loss due to friction in the chain of transmission from the motor to the axis. Each part losses are defined as a sum of three terms, one constant, another depending only on the speed and the last depending on the torque transmitted. The robot joint kinematic chain is modeled with three elements: the motor, the gearbox and a rotational joint at the leg. The results show a good adequacy between measurement and simulation with the proposed identification method in comparison with a classic least square identification method. Copyright (C)2005 IFAC
\end{abstract}

Keywords: friction modeling, dynamic model identification, physical modeling, parameter estimation, Harmonic Drive gearbox, biped robot.

\section{INTRODUCTION}

Works on the control of certain robots, in particular bipeds, require thorough preliminary studies in simulation, precise modeling and identification. A characteristic of the robots with legs lies in the alternation of the phases of support and swinging, characterized by low speeds and strong loads for the first, high speeds and weak loads for the second. This alternation between two operating modes under even opposite very different conditions makes the order very sensitive to the effects of friction. As for any mechanical system, frictions are difficult to model and many works are pub-

\footnotetext{
1 We thank all the participants of the group "Legged Robots" from the "Robéa" project of CNRS for their assistance and their participation in discussions which made it possible to advance this work. We thank more particularly Messrs S. Lydoire, P. Poignet and G. Buche which provided us certain experimental data.
}

lished on this subject (Armstrong (1988), Dupont (1990)). The difficulty to obtain a precise model comes owing from the fact that a great number of parts intervene in the transmission, in particular in the gear. The Rabbit robot was designed within the framework of a French national project as an experimental platform for the walk and race of biped robots. Its modeling and the identification of the parameters of the model form part of the objectives of the project. This paper presents suggestions for modeling and the results obtained by using an approach based on the decomposition of the kinematic chain in independent elements and on the analysis of the mechanical power flow which goes through these elements. This approach was already carried out by other authors (Pennestri et al. (1993), Abba et al. (1999), Filipoiu et al. (1996)), the characteristic of our work lies in its application to "Harmonic Drive" gearboxes. 


\section{FRICTION MODELING}

\subsection{Relation between speed and friction}

The relation between friction and speed is well modeled in the literature. One considers, as the fig. 1.(a) shows it, that friction comprises a principal term $F_{0}$, known as kinematic Coulomb friction $\left(F=F_{0}\right.$ at low speed $\left.\dot{q}_{0}\right)$, a term $F_{1}$, known as static Coulomb friction $\left(F=F_{0}+F_{1}\right.$ at null speed), as well as terms of viscous friction proportional to the speed and to its square. A traditional formulation (referring for example to Canudas et al. (1997)) is as follows:

$$
F=\left(F_{0}+F_{1} e^{-\left(\dot{q} / \dot{q}_{0}\right)^{2}}\right) \operatorname{sign}(\dot{q})+f_{2} \dot{q}+f_{3} \dot{q}|\dot{q}|,
$$

where $\dot{q}$ represents the speed.

The terms of friction $F_{0}$ and $F_{1}$ as well as the coefficients of viscous friction $f_{2}$ and $f_{3}$ are often regarded as constant. Friction is then a function of the speed $F=F(\dot{q})$. But this consideration conforms to reality only in certain cases of very simple machines (such as for example an electric motor) or in certain very specific cases of operation, with constant loads. However the majority of the mechanisms operates under variable loads. It is the case of the robots in general and more particularly of the legged robots because the legs are either carrying the structure (support phase) or are alternatively suspended there (swinging phase). Under variable loads (see fig. 1.(b)), the friction depends at the same time on the speed and on the load: $F=F(\Gamma, \dot{q})$ where $\Gamma$ represents the load. In the majority of the cases, the influence of the load is more important besides than that of the speed, in the sense that the increment of friction on the effective field of load (at constant speed) is higher than that which corresponds to the speed range used (at constant load). If the relations friction-speed were the subject of many studies in the literature, those as Gogoussis et al. (1987) concerning the relation friction-load-speed are rarer.

In this paper, we propose a simple model, in which the terms of Coulomb friction are governed by a function closely connected to the load, $F_{1}=c F_{0}$ and $F_{0}=F_{r}+\mu|\Gamma|$ where $F_{r}$ is a term of constant friction and $\mu$ the proportionality factor compared to the load $\Gamma$, which will be defined with accuracy later on. Thus, the general relation is obtained:

$$
\begin{array}{r}
F=\left(F_{r}+\mu|\Gamma|\right)\left(1+c e^{-\left(\frac{\dot{q}}{q_{0}}\right)^{2}}\right) \operatorname{sign}(\dot{q}) \\
+f_{2} \dot{q}+f_{3} \dot{q}|\dot{q}|
\end{array}
$$

The static friction is felt at the start but influences little the general working. Indeed, when the direction of the movement is reversed, the speed is canceled without relieving the materials. So the

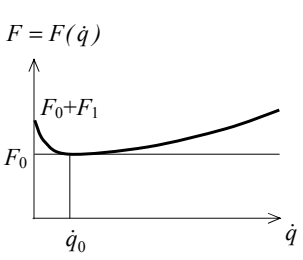

(a)

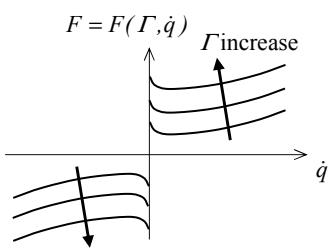

(b)
Fig. 1. Friction terms versus load and speed

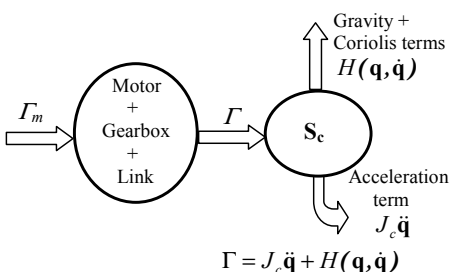

(a)

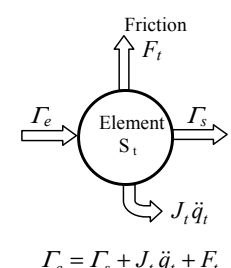

(b)
Fig. 2. Flow diagram of torque transmission

Stribeck effect can be neglected $\left(F_{1}=0\right.$ thus $c=0)$. In addition, the quadratic viscous friction effect appears little in the robot transmissions, therefore the coefficient $f_{3}$ can be neglected. By setting out $f_{2}=f$, one obtains the relation $F=\left(F_{r}+\mu|\Gamma|\right) \operatorname{sign}(\dot{q})+f \dot{q}$.

\subsection{Elements of transmission of a robot axis}

In a robot, frictions appear in the various elements of transmission of each axis. One can distinguish three of them: the motor, the gearbox elements (gears or screw and nut mechanisms) and finally joining elements (bearings or smooth bearings). Figure 2.(a) shows that the motor torque $\Gamma_{m}$ is transformed by all these elements of transmission into joint torque $\Gamma$, which puts the body $S_{c}$ moving (quantity of acceleration $\mathbf{J}_{c} \ddot{\mathbf{q}}$ ) after having overcome gravity, and the centrifugal and Coriolis effects $(\operatorname{term} H)$ :

$$
\Gamma(\mathbf{q}, \dot{\mathbf{q}}, \ddot{\mathbf{q}})=\mathbf{J}_{c} \ddot{\mathbf{q}}+H(\mathbf{q}, \dot{\mathbf{q}}),
$$

where $\mathbf{q}$ represents the vector of the articular positions of the robot and $\mathbf{J}_{c}$ the line vector of the global inertia matrix corresponding to the considered axis of the robot. By so organizing the axis of the robot, as that is proposed in Gogoussis et al. (1993), all frictions are set off upstream the joint torque. Now let us consider a mechanical element $S_{t}$ of the transmission, wherever it is, located between the motor and the robot axis. As figure 2.(b) suggests, the input torque $\Gamma_{e}$ is different from the output torque $\Gamma_{s}$, because of the inertia $J_{t}$ of the element and of the frictions $F_{t}$, thus $\Gamma_{e}=\Gamma_{s}+J_{t} \ddot{q}_{t}+F_{t}$.

The final relation between input and output torques is obtained by replacing in the preceding relation $F_{t}$ by : $\left(F_{r}+\mu\left|\Gamma_{s}\right|\right) \operatorname{sign}\left(\dot{q}_{t}\right)+f \dot{q}_{t}$, what gives $\Gamma_{e}=\left(1+\mu \operatorname{sign}\left(\Gamma_{s} \dot{q}_{t}\right)\right) \Gamma_{s}+J_{t} \ddot{q}_{t}+$ $F_{r} \operatorname{sign}\left(\dot{q}_{t}\right)+f \dot{q}_{t}$. It is possible to justify very 


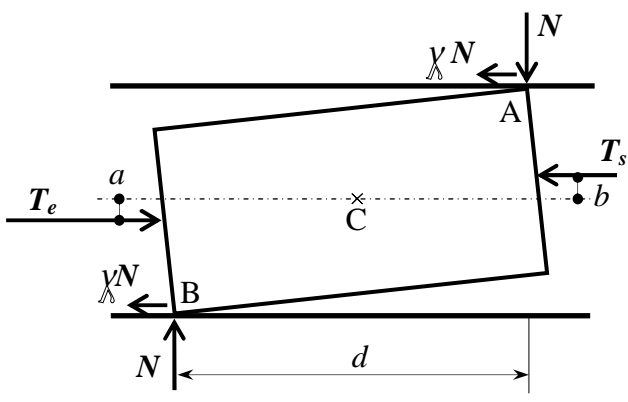

Fig. 3. Meaningful friction modeling in a prismatic joint

simply why the affine relation of friction is written according to the output torque $\Gamma_{s}$. This is the object of the following section.

\subsection{A simple meaningful study : friction in a prismatic joint}

Let us consider a slide connection made up of a parallelepipedic block with the length $d$ slipping between two plans, as it is drawn in figure 3 . The input force $T_{e}$ (resp. the output $T_{s}$ ) is offset of a distance $a$ (resp. $b$ ) compared to the slide axis (of center $C$ ). The normal efforts $N$ appearing at the contact points $\mathrm{A}$ and $\mathrm{B}$ are at the origin of tangential Coulomb friction forces, $T=\nu N$. The total friction force is worth $F=2 T$. The fundamental principle of statics results in $F=$ $2 \nu\left(a T_{e}+b T_{s}\right) / d$.

Let us examine two cases now: if $a=0$, then $F=\mu T_{s}$ (Case 1 , with $\left.\mu=2 \nu b / d\right)$, and if $b=0$, then $F=\mu T_{e}$ (Case 2 , with $\mu=$ $2 \nu a / d)$. Let us calculate in both cases the frontto-back transmission efficiency (the input involves the output, $\left.\left|T_{e}\right|>\left|T_{s}\right|\right)$ and the front-to-rear transmission efficiency (the output involves the input, $\left.\left|T_{e}\right|<\left|T_{s}\right|\right)$. In the first case, $\eta_{m}=1 /(1+$ $\mu$ ) and $\eta_{r}=1-\mu$, while it is the opposite in the second case. Thus, $\eta_{m}>\eta_{r}$ in the first case and $\eta_{m}<\eta_{r}$ in the second case. The efficiencies measured by the manufacturers of mechanical components are always in conformity with the first case, which justifies the choice to write the affine function of friction according to $\Gamma_{s}$.

A second justification can be deduced from the small example above. Indeed, in reality, when the characteristics of the industrial components are examined, it is noted on the one hand that the efficiencies decrease very appreciably at the same time as $\Gamma_{s}$, and on the other hand that $\eta_{r}$ is not defined for the values $\Gamma_{s}$ lower than a level of reversibility. This behavior is taken into account in our model thanks to the term of constant friction $F_{r}$. Indeed, one then obtains the following expressions which are in conformity with the industrial data:

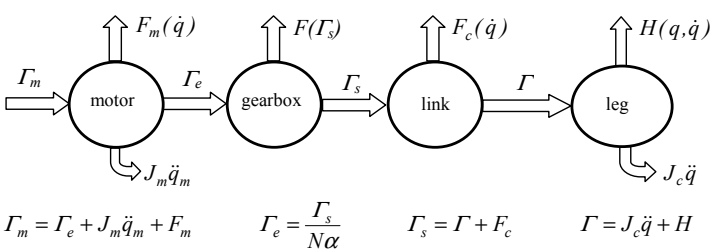

Fig. 4. Flow diagram of the studied joint

$$
\eta_{m}=\frac{1}{1+\mu+\frac{F_{r}}{T_{s}}}, \quad \text { and } \quad \eta_{r}=1-\mu-\frac{F_{r}}{T_{s}} .
$$

\section{MODELING OF A JOINT OF THE BIPED ROBOT RABBIT}

The biped robot Rabbit consists of two legs provided with knees joints and a trunk of important mass connected to the legs by the hip joints. The robot does not have feet. We will detail in this paragraph the modeling of the axis of a knee suspended freely in space. In this case, there is no contact with the ground and thus no efforts exerted on the free part of the tibia. The leg of the Rabbit robot consists of an aluminum body provided in its end with a wheel placed in an orthogonal plan with the walk direction (see Robot à pattes (1999)). The kinematic chain of the articulation of the knee is made up of a D.C. motor which actuates via a molded notch belt the input shaft of an "Harmonic Drive" gearbox. The dynamic spline of the gear is directly coupled with the leg of the robot.

The connection pivot between the thigh and the tibia is carried out by a double bearing integrated in the Harmonic Drive gearbox. The elements of transmission described above are represented on the flow chart of figure 4 . When the foot of the robot is not in contact with the ground and that only the considered axis is actuated, the articular couple $\Gamma$ applied to the leg is given by the equation (3), in which the term $H$ depends only on the position $q$ and the first moment of inertia $m l$ of the leg, that is to say $\Gamma(q, \dot{q}, \ddot{q})=J_{c} \ddot{q}+m l \sin (q)$. The torque $\Gamma_{s}$ at gearbox output can be written:

$$
\Gamma_{s}=\Gamma(q, \dot{q}, \ddot{q})+F_{c}(\dot{q}),
$$

where $F_{c}(\dot{q})$ represents the friction torque in the connection thigh-tibia.

Taking into account the theoretical elements explained previously, the equations of modeling of the reducer are given by:

$$
\dot{q}=\frac{\dot{q}_{m}}{N}, \Gamma_{s}=\frac{N \Gamma_{e}}{1+\mu_{i} \operatorname{sign}\left(\Gamma_{s} \dot{q}\right)}=\alpha_{i} N \Gamma_{e}
$$

where $\dot{q}_{m}$ represents the motor speed, $\frac{1}{N}$ represents the gearbox ratio, $\alpha_{i}$ represents the torque transfer coefficient and $\Gamma_{e}$ represents the gearbox input torque. 
The value of the coefficient $\alpha_{i}$ depends on the direction of the power transmission in the gearbox. We have two values to distinguish, one corresponding to the transfer of output from the motor towards the axis which will be noted $\alpha_{1}$ and the other corresponding to the transfer in opposite direction noted $\alpha_{2}$.

The dynamic behavior of the driving axis is given by the equation:

$$
\Gamma_{m}=J_{m} \ddot{q}_{m}+F_{m}\left(\dot{q}_{m}\right)+\Gamma_{e},
$$

where $\Gamma_{m}, \dot{q}_{m}$ et $\ddot{q}_{m}$ respectively represent the torque, the angular velocity and the angular acceleration of the motor, $J_{m}$ corresponds to the inertia of the motor and of the gearbox input axis and $F_{m}\left(\dot{q}_{m}\right)$ is the torque of the friction forces on the motor.

Initially and in order to simplify our presentation, we used a relatively simple friction model. A more precise modeling is implemented which integrates non symmetrical terms of friction for the two directions of rotation. We then have the expressions of the friction torques $F_{c}(\dot{q})$ and $F_{m}\left(\dot{q}_{m}\right)$ which are put in the form:

$$
\begin{aligned}
& F_{c}(\dot{q})=\frac{C_{c_{1}}}{2}(\operatorname{sign}(\dot{q})+1)+\frac{C_{c_{2}}}{2}(\operatorname{sign}(\dot{q})-1)+f_{c} \dot{q} \\
& F_{m}\left(\dot{q}_{m}\right)=\frac{C_{m_{1}}}{2}\left(\operatorname{sign}\left(\dot{q}_{m}\right)+1\right) \\
& \quad+\frac{C_{m_{2}}}{2}\left(\operatorname{sign}\left(\dot{q}_{m}\right)-1\right)+f_{m} \dot{q}_{m},
\end{aligned}
$$

with $C_{c_{1}}$ and $C_{c_{2}}$ terms of Coulomb friction in direct and opposite direction for the connection of the axis of the leg and $C_{m_{1}}$ and $C_{m_{2}}$ similar terms for the motor side.

\section{METHOD FOR THE IDENTIFICATION OF THE ROBOT AXIS}

We find in the literature many works on the identification of the model of a mechanical system. The linear quadratic methods are recommended when the model of the system is linear in relation to the coefficients to identify and that the signal of excitation is sufficiently informative.

\subsection{Identification with least square method}

The least square method of identification of which one thinks initially, requires the possibility for the equations characterizing the model of the physical system to be written in a linear way according to the parameters to identify. In our case, it is necessary to rewrite the equation (6) in the form:

$$
\Gamma_{s}=\alpha_{m} N \Gamma_{e},
$$

where $\alpha_{m}$ represents the average transfer coefficient of the gearbox, the definitions of the other variables remaining unchanged. The control variable being the motor current $I_{m}$, the relation

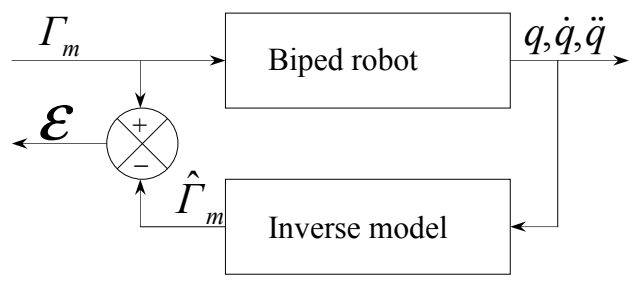

Fig. 5. Direct identification method

$\Gamma_{m}=k_{e} I_{m}$ is well-known $\left(k_{e}=0,32\right.$ given by the manufacturer). The relations (5) and (7) to (9) give, all calculations made, the following equation:

$$
\begin{array}{r}
\Gamma_{m}=J_{t} \ddot{q}_{m}+f_{t} \dot{q}_{m}+C_{t} \operatorname{sign}\left(\dot{q}_{m}\right) \\
+\Delta C_{t}+\frac{m l}{\alpha_{m} N} \sin \left(\frac{q_{m}}{N}\right),
\end{array}
$$

with

$$
\begin{aligned}
J_{t} & =J_{m}+\frac{J_{c}}{\alpha_{m} N^{2}}, \quad f_{t}=f_{m}+\frac{f_{c}}{\alpha_{m} N^{2}}, \\
C_{t} & =\frac{C_{m_{1}}+C_{m_{2}}}{2}+\frac{C_{c_{1}}+C_{c_{2}}}{2 \alpha_{m} N}
\end{aligned}
$$

and $\Delta C_{t}=\frac{C_{m_{1}}-C_{m_{2}}}{2}+\frac{C_{c_{1}}-C_{c_{2}}}{2 \alpha_{m} N}$,

respectively the global inertia, the coefficient of global viscous friction, the Coulomb friction and the difference of Coulomb friction brought back on the driving axis. By applying the traditional least square method, we obtain the numerical values of the identifiable coefficients given in table 1 . We will comment on these values thereafter.

\begin{tabular}{|c|c|}
\hline \multicolumn{2}{|c|}{ Global parameters on the motor side } \\
\hline$J_{t}=4,710^{-4} \mathrm{~kg} . \mathrm{m}^{2}$ & $f_{t}=6,510^{-3} \mathrm{Nm} . \mathrm{s}$ \\
\hline$C_{t}=0,203 \mathrm{Nm}$ & $\Delta C_{t}=-0,0025 \mathrm{Nm}$ \\
\hline
\end{tabular}

Table 1. Identification results by least squares for a signal with $1 \mathrm{~Hz}$.

\subsection{Direct identification method by minimization of the input error}

One can easily note that the model of behavior obtained starting from the equations (5) to (9) is nonlinear according to the parameters. It is then important to know if this model is identifiable (see Tunali et al. (1987)). The equation (5) explicitly giving the opposite model of the robot, we define the function $\hat{\Gamma}_{m}(\hat{\theta})(\hat{\theta}$ is the vector of parameters $)$ representing the inverse model of the system. The error criterion $\Delta$ is defined by the quadratic error between the motor torque and the function $\hat{\Gamma}_{m}(\hat{\theta})$ as shown in the figure 5 .

Let us define:

$$
\Delta=\left(\Gamma_{m}-\hat{\Gamma}_{m}\right)^{T}\left(\Gamma_{m}-\hat{\Gamma}_{m}\right)
$$

with $\Gamma_{m}$ the measured torque and the torque $\hat{\Gamma}_{m}$ obtained by the inverse model according to measurements $q_{m}, \dot{q}_{m}, \ddot{q}_{m}$ and of the parameters.

For two different values $\hat{\theta}_{a}=\left(\theta_{1}, \theta_{2}, \alpha_{1}, \alpha_{2}\right)$ and $\hat{\theta}_{b}=\left(\theta_{1}, \frac{\theta_{2}}{2}, 2 \alpha_{1}, 2 \alpha_{2}\right)$, we obtain the same values 
of the function $\Delta$. The whole of the physical parameters of the model are thus not identifiable.

The equations (5) to (9) allow to obtain the equation of the torque $\hat{\Gamma}_{m}$ :

$$
\hat{\Gamma}_{m}=\psi \hat{\Theta}_{1}+\operatorname{sign}\left(\dot{q}_{m}\right) \otimes\left|\psi \hat{\Theta}_{2}\right|,
$$

with the vector $\psi=\left[\ddot{q}_{m} \dot{q}_{m} \operatorname{sign}\left(\dot{q}_{m}\right) 1 \sin \left(\frac{q_{m}}{N}\right)\right]^{T}$ and the vectors of parameters to identify $\hat{\Theta}_{1}=$ $\left[\begin{array}{lllll}\theta_{1} & \theta_{2} & \theta_{3} & \theta_{4} & \theta_{5}\end{array}\right]^{T}$ and $\hat{\Theta}_{2}=\left[\begin{array}{lllll}\theta_{6} & \theta_{7} & \theta_{8} & \theta_{9} & \theta_{10}\end{array}\right]^{T}$. The symbol $\otimes$ represents the inner product of two vectors and the symbol $|V|$ represents a vector made up of the absolute values of the elements of $V$. The expressions of the parameters according to the values defined previously are: $\theta_{1}=J_{m}+\frac{\lambda_{1} J_{c}}{N^{2}}$, $\theta_{2}=f_{m}+\frac{\lambda_{1} f_{c}}{N^{2}}, \theta_{3}=\frac{C_{m_{1}}+C_{m_{2}}}{2}+\frac{\lambda_{1}\left(C_{c_{1}}+C_{c_{2}}\right)}{2 N}, \theta_{4}=$ $\frac{C_{m_{1}}-C_{m_{2}}}{2}+\frac{\lambda_{1}\left(C_{c_{1}}-C_{c_{2}}\right)}{2 N}, \theta_{5}=\frac{\lambda_{1} m l}{N}, \theta_{6}=\frac{\lambda_{2} J_{c}}{N^{2}}$, $\theta_{7}=\frac{\lambda_{2} f_{c}}{N^{2}}, \theta_{8}=\frac{\lambda_{2}\left(C_{c_{1}}+C_{c_{2}}\right)}{2 N}, \theta_{9}=\frac{\lambda_{2}\left(C_{c_{1}}-C_{c_{2}}\right)}{2 N}$, $\theta_{10}=\frac{\lambda_{2} m l}{N}$.

The identification of the system then amounts seeking the vectors of parameters $\hat{\Theta}_{1}$ and $\hat{\Theta}_{2}$ which minimizes the error function $\Delta$ for the whole of measurement. The gradient of the error function for each vector of parameters is given by:

$$
\begin{aligned}
& \frac{\partial \Delta}{\partial \hat{\Theta}_{1}}=-2 \frac{\partial \hat{\Gamma}_{m}^{T}}{\partial \hat{\Theta}_{1}}\left(\Gamma_{m}-\hat{\Gamma}_{m}\right), \\
& \frac{\partial \Delta}{\partial \hat{\Theta}_{2}}=-2 \frac{\partial \hat{\Gamma}_{m}^{T}}{\partial \hat{\Theta}_{2}}\left(\Gamma_{m}-\hat{\Gamma}_{m}\right) .
\end{aligned}
$$

The derivatives of the torque $\hat{\Gamma}_{m}$ are given by:

$$
\begin{aligned}
& \frac{\partial \hat{\Gamma}_{m}^{T}}{\partial \hat{\Theta}_{1}}=\psi, \\
& \frac{\partial \hat{\Gamma}_{m}^{T}}{\partial \hat{\Theta}_{2}}=\operatorname{sign}\left(\dot{q}_{m}\right) \otimes\left(\psi \hat{\Theta}_{2}\right) \circledast \psi=\psi_{1}\left(\hat{\Theta}_{2}\right),(17
\end{aligned}
$$

where the symbol $\circledast$ represents the operation between a vector and a matrix. The result of this operation is a matrix whose columns are obtained by the inner product of the vector and of each column of the matrix.

After calculations, the equation (14) makes to calculate the vector of the parameters $\hat{\Theta}_{1}$ :

$$
\hat{\Theta}_{1}=\left(\psi^{T} \psi\right)^{-1} \psi^{T}\left[\Gamma_{m}-\operatorname{sign}\left(\dot{q}_{m}\right) \otimes\left|\psi \hat{\Theta}_{2}\right|\right] .
$$

The equation (15) becomes:

$$
\psi_{1}\left(\hat{\Theta}_{2}\right)\left[\Gamma_{m}-\psi \hat{\Theta}_{1}-\operatorname{sign}\left(\dot{q}_{m}\right) \otimes\left|\psi \hat{\Theta}_{2}\right|\right]=0 .
$$

By replacing $\hat{\Theta}_{1}$ in (19), one obtains an equation which cannot be solved in explicit form, but only numerically.

By using the same data of measurement that in the paragraph 4.1 , we obtain thus the values of the vector $\hat{\Theta}_{2}$ and by applying the equation (18) the values of $\hat{\Theta}_{1}$. Tests not described here for lack

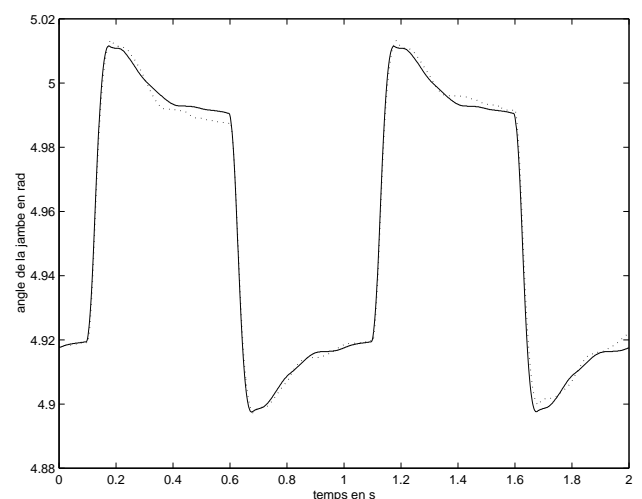

Fig. 6. Comparison between measurement (solid line) and simulation (dotted) for $1 \mathrm{~Hz}$ signal of place make it possible to determine the values $\lambda_{1}$ and $\lambda_{2}$. We can then calculate the values of the whole of the physical model parameters which are given in the table 2 .

\begin{tabular}{|c|c|c|}
\hline \multirow{2}{*}{ Motor } & $J_{m}=3.210^{-4} \mathrm{~kg} \mathrm{~m}^{2}$ & $f_{m}=2.410^{-3} \mathrm{Nm} \mathrm{s}$ \\
\cline { 2 - 3 } & $C_{m_{1}}=0.106 \mathrm{Nm}$ & $C_{m_{2}}=0.100 \mathrm{Nm}$ \\
\hline Gearbox & $\alpha_{1}=0.75$ & $\alpha_{2}=1.31$ \\
\hline \multirow{3}{*}{ Leg } & $J_{c}=0.24 \mathrm{~kg} \mathrm{~m}^{2}$ & $f_{c}=6.9 \mathrm{Nm} \mathrm{s}$ \\
& $C_{c_{1}}=4.51 \mathrm{Nm}$ & $C_{c_{2}}=4.75 \mathrm{Nm}$ \\
& $m l=0.58 \mathrm{~kg} \mathrm{~m}$ & \\
\hline
\end{tabular}

Table 2. Identification results by input error method for a signal with $1 \mathrm{~Hz}$.

Our experiment on this method enables us to conclude that it is sensitive to the choice of filters. The results obtained for the friction and the first order momentum are not very precise. On the other hand, the values of inertia are given with a good precision.

\subsection{Method of recursive identification}

The method of identification used in this case is a method known as recursive. This method first of all consists in transcribing the equations seen previously in a simulation software. The measurements taken on the robot are regarded as entries of the model. Simulation provides the simulated value of the swing angle of the leg noted $q_{c}$. From this signal and the measurement of the real angle of the leg, we can define a criterion of $\left\|q-q_{c}\right\|$ error which will depend only on the parameters of the model and on the agreement of the functions of the model with their exact expressions.

The experimental tests carried out on the Rabbit robot made it possible to record the evolution of the angles of the motor and joint axes for an alternative excitation in torque imposed by the motor. These measurements are applied in entry of a simulation program under Matlab-Simulink. We carry out then a loop of optimization in order to reduce the difference between the simulated and the measured articular angles. This loop 
of optimization uses the routine of optimization under constraint and multivariable fmincon also available under Matlab. The square form signal is applied to the control input of the knee axis, which imposes the current $I_{m}$. The motor torque is proportional to the value $I_{m}$ according to the relation $\Gamma_{m}=k_{e} I_{m}$. The coefficient $k_{e}$ is given by the manufacturer and is known with precision, thus it does not need to be identified. Figure 6 shows the optimum result obtained by simulation with the model suggested. There is a good agreement between the two curves. The result of optimization gives the numerical values indicated in table 3 of the various coefficients of the model.

\begin{tabular}{|c|c|c|}
\hline \multirow{2}{*}{ Motor } & $J_{m}=3.5610^{-4} \mathrm{~kg} \mathrm{~m}^{2}$ & $f_{m}=4.010^{-3} \mathrm{Nm} \mathrm{s}$ \\
\cline { 2 - 3 } & $C_{m_{1}}=0.087 \mathrm{Nm}$ & $C_{m_{2}}=0.091 \mathrm{Nm}$ \\
\hline Gearbox & $\alpha_{1}=0.7432$ & $\alpha_{2}=1.408$ \\
\hline \multirow{3}{*}{ Leg } & $J_{c}=0.206 \mathrm{~kg} \mathrm{~m}^{2}$ & $f_{c}=3.12 \mathrm{Nm} \mathrm{s}$ \\
& $C_{c_{1}}=5.03 \mathrm{Nm}$ & $C_{c_{2}}=5.15 \mathrm{Nm}$ \\
& $m l=0.42 \mathrm{~kg} \mathrm{~m}$ & \\
\hline
\end{tabular}

Table 3. Identification results by recursive method for a signal with $1 \mathrm{~Hz}$.

\subsection{Comments on the results}

These numerical values of identification can be compared with those provided by the manufacturers (of the motor and the gearbox) or with the theoretical values calculated by the CAD system with regard to the mechanical parts of the leg. The motor is of type Parvex RS420J, and the gearbox Harmonic Drive of type HFUS-2UH, size 25, ratio $N=50$.

The result obtained for the moment of inertia $J_{m}$ is close to the sum "inertia of the rotor plus inertia of the input shaft of the gearbox", which is worth $3.3210^{-4} \mathrm{~kg} \mathrm{~m}^{2}$. The moment of inertia $J_{m}$ increased with the moment of inertia $J_{c}$ brought back to the motor side $J_{c} N^{-2}$ is worth $4.3810^{-4} \mathrm{~kg} \mathrm{~m}^{2}$, which is comparable with the globally evaluated moment of inertia $J_{t}$. The coefficient of viscous friction $f_{m}$ increased with the coefficient $f_{c}$ brought back to the motor side is worth $5.310^{-3} \mathrm{Nm} \mathrm{s}$, which is rather close to the total viscous coefficient $f_{t}$. The term of constant friction $C_{m_{1}}$ increased with $C_{c_{1}}$ brought back to the motor side $\left(C_{c_{1}} N^{-1}\right)$ is worth 0.188 $\mathrm{Nm}$, which is comparable to the term $C_{t}$ globally evaluated. A relatively high part of the power is dissipated within the gearbox. It is important to evaluate the relevance of the $\alpha_{1}$ and $\alpha_{2}$ terms. By taking into account the equations (4) and (6), one obtains a driving efficiency of 0.692 and one receiving efficiency of 0.607 , which is completely in conformity with the corresponding data of Harmonic Drive under the average conditions of the experiment. Lastly, the first moment of the leg $m l$ is identified with a quasi-identical value to that calculated in CAD, namely $0.41 \mathrm{~kg} \mathrm{~m}$.

\section{CONCLUSION}

This work proposes a method of decomposition of the friction model in a kinematic chain of a robot axis in three terms, one constant, the other depending only on the speed of the axis and the last of the torque transmitted to the axis. The result of such a modeling makes it possible to differentiate the friction parameters from the motor axis, the gearbox and the connection of the output axis. The identification of these parameters is more complex, the new model being nonlinear in these parameters. A method of recursive identification is used and makes it possible to obtain the whole of the parameters of the model. The results of simulation compared with the experimental data obtained on the robot show a good correlation and validate the method suggested.

\section{REFERENCES}

Abba, G. and Chaillet, N. 1999. Robot Dynamic Modeling Using a Power Flow Approach with Application to Biped Locomotion. Autonomous Robots, 6:39-52.

Armstrong, B. 1988. Friction: Experimental Determination, Modeling and Compensation. In Proc. of IEEE Int. Conf. on Robotics and Automation, Philadelphia, PN, USA, April 24-29, vol. 3, pp. 1422-7.

Canudas-de-Wit, C. and Lischinsky, P. 1997. Adaptative Friction Compensation with Partially Known Dynamic Friction Model. Int. J. of Adapt. Control and Signal Proc., 11: 65-80.

Dupont, P.E. 1990. Friction modeling in dynamic robot simulation. In Proceedings of the IEEE Int. Conf. on Robotics and Automation, Cincinnati, USA, 13-18 May 1990, vol.3, pp. 1370-6.

Filipoiu, I.D., Taille, R. and Grecu, E. 1996. Computing the Efficiency of Toothed Gearings using Power Losses. In Proc. of $1^{\text {st }}$ Congress on Mechatronics, Besançon, France, 1-3 October, vol.1, pp. 442-6.

Gogoussis, A. and Donath, M. 1987. Coulomb Friction Joint and Drive Effects in Robot Mechanisms. In Proc. of the IEEE Int. Conf. on Rob. and Automation, Raleigh, NC, pp. 828-36.

Gogoussis, A. and Donath, M. 1993. Determining the Effects of Coulomb Friction on the Dynamics of Bearings and Transmissions in Robot Mechanisms. J. of Mechanical Design, 115(1): 231-40.

Pennestri, E. and Freudenstein, F. 1993. The Mechanical Efficiency of Epicyclic Gear Trains. Journal of Mechanical Design, 115(3): 645-51.

Robot à pattes, 1999. Photos du robot Rabbit, projet Robéa du CNRS, In site www.lagb29.ensieg.inpg.fr.

Tunali, E.T. and Tarn, T.J. 1987. New results for identifiability of nonlinear systems. IEEE Trans. Automatic Control, vol. AC-32:146-154. 